

\title{
Optimized Schwarz methods for the time-harmonic Maxwell equations with damping
}

Mohamed El Bouajaji, Victorita Dolean, Martin Gander, Stephane Lanteri

\section{To cite this version:}

Mohamed El Bouajaji, Victorita Dolean, Martin Gander, Stephane Lanteri. Optimized Schwarz methods for the time-harmonic Maxwell equations with damping. 2011. hal-00637822

\section{HAL Id: hal-00637822 \\ https://hal.science/hal-00637822}

Preprint submitted on 3 Nov 2011

HAL is a multi-disciplinary open access archive for the deposit and dissemination of scientific research documents, whether they are published or not. The documents may come from teaching and research institutions in France or abroad, or from public or private research centers.
L'archive ouverte pluridisciplinaire HAL, est destinée au dépôt et à la diffusion de documents scientifiques de niveau recherche, publiés ou non, émanant des établissements d'enseignement et de recherche français ou étrangers, des laboratoires publics ou privés. 


\title{
OPTIMIZED SCHWARZ METHODS FOR THE TIME-HARMONIC MAXWELL EQUATIONS WITH DAMPING
}

\author{
M. EL BOUAJAJI* V. DOLEAN ${ }^{\dagger}$ M.J. GANDER ${ }^{\ddagger}$ AND S. LANTERI*
}

\begin{abstract}
In a previous paper, two of the authors have proposed and analyzed an entire hierarchy of optimized Schwarz methods for Maxwell's equations both in the time-harmonic and time-domain case. The optimization process has been perfomed in a particular situation where the electric conductivity was neglected. Here, we take into account this physical parameter which leads to a fundamentally different analysis and a new class of algorithms for this more general case. From the mathematical point of view, the approach is different, since the algorithm does not encounter the pathological situations of the zero-conductivity case and thus the optimization problems are different. We analyze one of the algorithms in this class in detail and provide asymptotic results for the remaining ones. We illustrate our analysis with numerical results.
\end{abstract}

Key words. Schwarz algorithms, optimized transmission conditions, Maxwell's equations

AMS subject classifications. $65 \mathrm{M} 55,65 \mathrm{~F} 10,65 \mathrm{~N} 22$

1. Introduction. Finding robust solvers for indefinite problems, such as Helmholtz and the time-harmonic version of Maxwell's equations, has always been a challenging research topic. From the pioneering work of Desprès [5], where the first provably convergent domain decomposition algorithm for Helmholtz equations was proposed and then extended to Maxwell's equations in [6], other studies followed with the declared purpose of improving the performance of these algorithms. The first attempts to obtain better algorithms for this kind of equations were given in [4] and then [3], where the first inspiring ideas of optimized Schwarz methods can be found. Then, in [15] and in particular in [14], the advantange of the optimization process was used at its best for the Helmholtz equations to design an algorithm without overlap; for the second order Maxwell system see [1]. But, for the first time, an entire hierarchy of optimized transmission conditions for the first order Maxwell's equations was given in [8], with complete asymptotic results. Applications on real life problems using a Discontinunous Galerkin method can be found in [9, 10]. For finite-element based non-overlapping and non-conforming domain decomposition methods for the computation of multiscale electromagnetic radiation and scattering problems we refer to $[16,18,17]$.

This paper is organized as follows: in Section 2, we introduce the mathematical formulation of the problem to be solved, and in Section 3, the classical and optimized Schwarz algorithms are presented. These algorithms are analyzed by computing their convergence factor in Fourier space in Section 4. The main part of the paper and the core of this study is given in Section 5, where an optimization problem is solved for one of the optimized Schwarz methods in all detail, and asymptotic results are given for all optimized Schwarz algorithms considered in this paper.

*INRIA SOPHIA ANTIPOLIS-MÉDITERRANÉE, 06902 SOPHIA ANTIPOLIS CEDEX, FRANCE.

${ }^{\dagger}$ UNIV. DE NICE SOPHIA-ANTIPOLIS, LABORATOIRE J.-A. DIEUDONNÉ, NICE,

FRANCE. DOLEAN@UNICE.FR

¥SECTION DE MATHÉMATIQUES, UNIVERSITÉ DE GENÈVE, CP 64, 1211 GENÈVE, MARTIN.GANDER@MATH.UNIGE.CH

${ }^{*}$ INRIA SOPHIA ANTIPOLIS-MÉDITERRANÉE, 06902 SOPHIA ANTIPOLIS CEDEX, FRANCE. 
2. Presentation of the problem. The system of Maxwell's equations describes mathematically the propagation of electromagnetic waves

$$
\varepsilon \frac{\partial \mathcal{E}}{\partial t}-\operatorname{curl} \mathcal{H}+\sigma \mathcal{E}=-\mathcal{J}, \quad \mu \frac{\partial \mathcal{H}}{\partial t}+\operatorname{curl} \mathcal{E}=0
$$

where $\mathcal{E}$ and $\mathcal{H}$ denote the electric and magnetic field, $\varepsilon$ is the electric permittivity and $\mu$ the magnetic permeability. Here $\sigma$ denotes the conductivity and $\mathcal{J}$ the applied current density.

We are interested in solving the time-harmonic Maxwell equations, which are obtained from their time-domain counterpart (2.1) by assuming that the electric field $\mathcal{E}$ and the magnetic field $\mathcal{H}$ follow a harmonic dependence on time (as a result of the time-harmonic dependence of the current density $\mathcal{J}(\boldsymbol{x}, t)=\mathcal{R} e(\boldsymbol{J}(\boldsymbol{x}) \exp (i \omega t)))$,

$$
\mathcal{E}(\boldsymbol{x}, t)=\mathcal{R} e(\boldsymbol{E}(\boldsymbol{x}) \exp (i \omega t)), \quad \mathcal{H}(\boldsymbol{x}, t)=\mathcal{R} e(\boldsymbol{H}(\boldsymbol{x}) \exp (i \omega t)),
$$

where the positive real parameter $\omega$ is the pulsation of the harmonic wave. The unknow complex-valued vector fields $\boldsymbol{E}$ and $\boldsymbol{H}$ are the solutions of the time-harmonic Maxwell's equations

$$
i \omega \varepsilon \boldsymbol{E}-\operatorname{curl} \boldsymbol{H}+\sigma \boldsymbol{E}=-\boldsymbol{J}, \quad i \omega \mu \boldsymbol{H}+\operatorname{curl} \boldsymbol{E}=\mathbf{0} .
$$

Equations (2.2) are solved in a bounded domain $\Omega$. On the boundary $\partial \Omega=\Gamma_{a} \cup \Gamma_{m}$, the following boundary conditions are imposed:

- a perfect electric conductor condition on $\Gamma_{m}: \boldsymbol{n} \times \boldsymbol{E}=0$,

- an impedance condition on $\Gamma_{a}: \mathcal{B}_{\boldsymbol{n}}(\boldsymbol{E}, \boldsymbol{H})=\mathcal{B}_{\boldsymbol{n}}\left(\boldsymbol{E}_{\text {inc }}, \boldsymbol{H}_{\text {inc }}\right)$,

where the vector $\left(\boldsymbol{E}^{\text {inc }}, \boldsymbol{H}^{\text {inc }}\right)$ represents an incident electromagnetic wave and $\boldsymbol{n}$ denotes the unit outward normal at any point of $\partial \Omega$. The impedance operator is given by

$$
\mathcal{B}_{\boldsymbol{n}}(\boldsymbol{E}, \boldsymbol{H})=\boldsymbol{n} \times \frac{\boldsymbol{E}}{Z}-\boldsymbol{n} \times(\boldsymbol{H} \times \boldsymbol{n}) .
$$

Note that this condition is equivalent to imposing Dirichlet conditions on the incoming characteristics if we consider the hyperbolic nature of the underlying time-dependent system, and can be seen also as an approximation of a transparent boundary condition.

3. Schwarz Methods for Maxwell's Equations. We introduce here a domain decomposition method based on a Schwarz algorithm. The computational domain $\Omega$ with boundary $\partial \Omega$ is partitioned into $N$ subdomains $\Omega_{i}, i=1, . ., N$, such that $\bar{\Omega}=\bigcup_{i=1}^{N} \bar{\Omega}_{i}$. We set $\Gamma_{i j}=\partial \Omega_{i} \cap \bar{\Omega}_{j} \backslash \partial \Omega$ where $\partial \Omega_{i}$ is the boundary of $\Omega_{i}$. For a simple decomposition of the domain into two subdomains (as shown in Figure 3.1), the classical Schwarz algorithm consists in computing $\left(\boldsymbol{E}^{j, n+1}, \boldsymbol{H}^{j, n+1}\right)$ from $\left(\boldsymbol{E}^{j, n}, \boldsymbol{H}^{j, n}\right)$, for $j=1,2$ by the iterative process

$$
\begin{aligned}
& -i \omega \varepsilon \boldsymbol{E}^{1, n}+\operatorname{curl} \boldsymbol{H}^{1, n}-\sigma \boldsymbol{E}^{1, n}=\boldsymbol{J} \quad \text { in } \Omega_{1}, \\
& i \omega \mu \boldsymbol{H}^{1, n}+\operatorname{curl} \boldsymbol{E}^{1, n}=\mathbf{0} \quad \text { in } \Omega_{1}, \\
& \mathcal{B}_{\boldsymbol{n}_{1}}\left(\boldsymbol{E}^{1, n}, \boldsymbol{H}^{1, n}\right)=\mathcal{B}_{\boldsymbol{n}_{1}}\left(\boldsymbol{E}^{2, n-1}, \boldsymbol{H}^{2, n-1}\right) \text { on } \Gamma_{12}, \\
& -i \omega \varepsilon \boldsymbol{E}^{2, n}+\operatorname{curl} \boldsymbol{H}^{2, n}-\sigma \boldsymbol{E}^{2, n}=\boldsymbol{J} \text { in } \Omega_{2} \text {, } \\
& i \omega \mu \boldsymbol{H}^{2, n}+\operatorname{curl} \boldsymbol{E}^{2, n}=\mathbf{0} \quad \text { in } \Omega_{2}, \\
& \mathcal{B}_{\boldsymbol{n}_{2}}\left(\boldsymbol{E}^{2, n}, \boldsymbol{H}^{2, n}\right)=\mathcal{B}_{\boldsymbol{n}_{2}}\left(\boldsymbol{E}^{1, n-1}, \boldsymbol{H}^{1, n-1}\right) \quad \text { on } \Gamma_{21} \text {. }
\end{aligned}
$$

For the classical Schwarz method, we see that at the interfaces (artificial boundaries between subdomains), characteristic information is exchanged. This type of 




FIG. 3.1. Domain decomposition into two subdomains



FIG. 3.2. Convergence factor $\rho_{\text {cla }}$ of the classical Schwarz method as a function of $|\boldsymbol{k}|$, for $L=0, \omega=2 \pi, \sigma=2$ and $\mu=\varepsilon=1$.

conditions have been proposed for the first time in [6] for the second order version of the time-harmonic Maxwell equations.

In the constant coefficient case, an estimate of the convergence factor of this algorithm has been obtained in [8] using Fourier analysis. The convergence factor (the reduction of the error between two successive interations) for each Fourier mode $\boldsymbol{k}$ is given by

$$
\rho_{c l a}(\boldsymbol{k}, \tilde{\omega}, \sigma, Z, L)=\left|\frac{\sqrt{|\boldsymbol{k}|^{2}-\tilde{\omega}^{2}+i \tilde{\omega} \sigma Z}-i \tilde{\omega}}{\sqrt{|\boldsymbol{k}|^{2}-\tilde{\omega}^{2}+i \tilde{\omega} \sigma Z}+i \tilde{\omega}} e^{-\sqrt{|\boldsymbol{k}|^{2}-\tilde{\omega}^{2}+i \tilde{\omega} \sigma Z} L}\right|,
$$

where $\tilde{\omega}=\omega \sqrt{\varepsilon \mu}$ denotes the adimensionalized frequency and $L$ is the overlap between domains. From this result, we see that if $\sigma>0$, the method converges also without overlap $(L=0)$. But for the high frequencies, the convergence factor is close to one and therefore the algorithm is not very efficient, see Figure 3.2.

The convergence can be improved by modifying the transmission conditions in 
the classical Schwarz algorithm (3.1), namely

$$
\begin{array}{rlrl}
-i \omega \varepsilon \boldsymbol{E}^{1, n}+\operatorname{curl} \boldsymbol{H}^{1, n}-\sigma \boldsymbol{E}^{1, n} & =\boldsymbol{J} & & \text { in } \Omega_{1}, \\
i \omega \mu \boldsymbol{H}^{1, n}+\operatorname{curl} \boldsymbol{E}^{1, n} & =\mathbf{0} & & \text { in } \Omega_{1}, \\
\left(\mathcal{B}_{\boldsymbol{n}_{1}}+\mathcal{S}_{1} \mathcal{B}_{\boldsymbol{n}_{2}}\right)\left(\boldsymbol{E}^{1, n}, \boldsymbol{H}^{1, n}\right) & =\left(\mathcal{B}_{\boldsymbol{n}_{1}}+\mathcal{S}_{1} \mathcal{B}_{\boldsymbol{n}_{2}}\right)\left(\boldsymbol{E}^{2, n-1}, \boldsymbol{H}^{2, n-1}\right) & \text { on } \Gamma_{12}, \\
-i \omega \varepsilon \boldsymbol{E}^{2, n}+\operatorname{curl} \boldsymbol{H}^{2, n}-\sigma \boldsymbol{E}^{2, n} & =\boldsymbol{J} & & \text { in } \Omega_{2}, \\
i \omega \mu \boldsymbol{H}^{2, n}+\operatorname{curl} \boldsymbol{E}^{2, n} & =\mathbf{0} & & \text { in } \Omega_{2}, \\
\left(\mathcal{B}_{\boldsymbol{n}_{2}}+\mathcal{S}_{2} \mathcal{B}_{\boldsymbol{n}_{1}}\right)\left(\boldsymbol{E}^{2, n}, \boldsymbol{H}^{2, n}\right) & =\left(\mathcal{B} \boldsymbol{n}_{2}+\mathcal{S}_{2} \mathcal{B}_{\boldsymbol{n}_{1}}\right)\left(\boldsymbol{E}^{1, n-1}, \boldsymbol{H}^{1, n-1}\right) & & \text { on } \Gamma_{21},
\end{array}
$$

where $\mathcal{S}_{j}, j=1,2$ are tangential, possibly pseudo-differential operators. For $\sigma=0$, different choices of $\mathcal{S}_{j}, j=1,2$ have been developed in [8] and lead to different optimized Schwarz algorithms for Maxwell's equations. In the next section, we develop different choices of $\mathcal{S}_{j}$ for the case $\sigma>0$. This leads to optimization problems which are very different from the case $\sigma=0$. The min-max problems can now have interior maxima, which complicates the mathematical analysis substantially, but their solution is necessary to obtain optimized transmission conditions in the case $\sigma>0$.

4. Convergence Analysis for Non-Zero Electric Conductivity. We present here an analysis of algorithm (3.2) for the case where the electric conductivity is nonzero, $\sigma>0$. In [8], it was proved that if the operators $\mathcal{S}_{l}, l=1,2$ have the Fourier symbol

$$
\mathcal{F}\left(\mathcal{S}_{l}\right)=\frac{1}{(\lambda+i \tilde{\omega})(\lambda+i \tilde{\omega}+\sigma Z)}\left[\begin{array}{cc}
k_{y}^{2}-k_{z}^{2}-\lambda \sigma Z & -2 k_{y} k_{z} \\
-2 k_{y} k_{z} & k_{z}^{2}-k_{y}^{2}-\lambda \sigma Z
\end{array}\right]
$$

where $\lambda=\sqrt{|\boldsymbol{k}|^{2}-\tilde{\omega}^{2}+i \tilde{\omega} \sigma Z}$, then the algorithm (3.2) converges in two iterations.

It was also shown that these symbols can be written in different forms,

$$
\begin{aligned}
\mathcal{F}\left(\mathcal{S}_{l}\right) & =\frac{1}{(\lambda+i \tilde{\omega})(\lambda+i \tilde{\omega}+\sigma Z)} M=\frac{1}{|\boldsymbol{k}|^{2}+\lambda \sigma Z} \frac{\lambda-i \tilde{\omega}}{\lambda+i \tilde{\omega}} M \\
& =\frac{1}{|\boldsymbol{k}|^{2}-\lambda \sigma Z} \frac{\lambda-i \tilde{\omega}-\sigma Z}{\lambda+i \tilde{\omega}+\sigma Z} M=(\lambda-i \tilde{\omega})(\lambda-i \tilde{\omega}-\sigma Z) \tilde{M}^{-1},
\end{aligned}
$$

where $M$ and $\tilde{M}$ are given by

$$
M=\left[\begin{array}{cc}
k_{y}^{2}-k_{z}^{2}-\lambda \sigma Z & -2 k_{y} k_{z} \\
-2 k_{y} k_{z} & k_{z}^{2}-k_{y}^{2}-\lambda \sigma Z
\end{array}\right], \tilde{M}=\left[\begin{array}{cc}
k_{y}^{2}-k_{z}^{2}+\lambda \sigma Z & -2 k_{y} k_{z} \\
-2 k_{y} k_{z} & k_{z}^{2}-k_{y}^{2}+\lambda \sigma Z
\end{array}\right],
$$

and $\lambda=\sqrt{|\boldsymbol{k}|^{2}-\tilde{\omega}^{2}+i \omega \sigma Z}$. This motivates different approximations of the transparent conditions in the context of optimized Schwarz methods. In the following we will denote by $M_{s}$ and $\tilde{M}_{s}$ the matrices $M$ and $\tilde{M}$ where we replaced the non-local operator $\lambda$ by a constant $s$.

COROLlary 4.1. For different symbols of the operators $S_{j}$ (which approximate the non local operators 4.1) we get the following convergence factors:

Algorithm 1 If $\mathcal{S}_{j}, j=1,2$, have the symbols

$$
\sigma_{j}=\mathcal{F}\left(\mathcal{S}_{j}\right)=0,
$$

then the Schwarz algorithm (3.2) has the convergence factor

$$
\rho_{1}(|\boldsymbol{k}|, \tilde{\omega}, \sigma, Z, L)=\left|\frac{\sqrt{|\boldsymbol{k}|^{2}-\tilde{\omega}^{2}+i \tilde{\omega} \sigma Z}-i \tilde{\omega}}{\sqrt{|\boldsymbol{k}|^{2}-\tilde{\omega}^{2}+i \tilde{\omega} \sigma Z}+i \tilde{\omega}} e^{-\sqrt{|\boldsymbol{k}|^{2}-\tilde{\omega}^{2}+i \tilde{\omega} \sigma Z} L}\right| .
$$

This algorithm is the classical Schwarz algorithm. 
Algorithm 2 If $\mathcal{S}_{j}, j=1,2$, have the Fourier symbols

$$
\sigma_{j}=\mathcal{F}\left(\mathcal{S}_{j}\right)=\frac{s-i \tilde{\omega}}{(s+i \tilde{\omega})\left(|\boldsymbol{k}|^{2}+s \sigma Z\right)} M_{s}, \quad s \in \mathbb{C},
$$

then the Schwarz algorithm (3.2) has the convergence factor

$$
\rho_{2}(|\boldsymbol{k}|, \tilde{\omega}, \sigma, Z, L, s)=\left|\left(\frac{\sqrt{|\boldsymbol{k}|^{2}-\tilde{\omega}^{2}+i \tilde{\omega} \sigma Z}-s}{\sqrt{|\boldsymbol{k}|^{2}-\tilde{\omega}^{2}+i \tilde{\omega} \sigma Z}+s}\right) e^{-\sqrt{|\boldsymbol{k}|^{2}-\tilde{\omega}^{2}+i \tilde{\omega} \sigma Z} L}\right| .
$$

Algorithm 3 If $\mathcal{S}_{j}, j=1,2$, have the Fourier symbols

$$
\sigma_{j}=\mathcal{F}\left(\mathcal{S}_{j}\right)=\frac{1}{|\boldsymbol{k}|^{2}-2 \tilde{\omega}^{2}+2 i \tilde{\omega} \sigma Z+(2 i \tilde{\omega}+\sigma Z) s} M_{s}, \quad s \in \mathbb{C},
$$

then (3.2) has the convergence factor

$$
\rho_{3}(|\boldsymbol{k}|, \tilde{\omega}, \sigma, Z, L, s)=\left|\left(\frac{\sqrt{|\boldsymbol{k}|^{2}-\tilde{\omega}^{2}+i \tilde{\omega} \sigma Z}-i \tilde{\omega}}{\sqrt{|\boldsymbol{k}|^{2}-\tilde{\omega}^{2}+i \tilde{\omega} \sigma Z}-i \tilde{\omega}}\right)\right| \rho_{2}(|\boldsymbol{k}|, \tilde{\omega}, \sigma, Z, L, s) .
$$

Algorithm 4 If $\mathcal{S}_{j}, j=1,2$, have the Fourier symbols

$$
\sigma_{j}=\mathcal{F}\left(\mathcal{S}_{j}\right)=\frac{s_{j}-i \tilde{\omega}}{\left(s_{j}+i \tilde{\omega}\right)\left(|\boldsymbol{k}|^{2}+s_{j} \sigma Z\right)} M_{s_{j}}, \quad s_{j} \in \mathbb{C},
$$

then the Schwarz algorithm (3.2) has the convergence factor

$$
\rho_{4}\left(|\boldsymbol{k}|, \tilde{\omega}, \sigma, Z, L, s_{1}, s_{2}\right)=\left|\prod_{l=1}^{2} \frac{\sqrt{|\boldsymbol{k}|^{2}-\tilde{\omega}^{2}+i \tilde{\omega} \sigma Z}-s_{l}}{\sqrt{|\boldsymbol{k}|^{2}-\tilde{\omega}^{2}+i \tilde{\omega} \sigma Z}+s_{l}} e^{-2 \sqrt{|\boldsymbol{k}|^{2}-\tilde{\omega}^{2}+i \tilde{\omega} \sigma Z} L}\right|^{\frac{1}{2}} .
$$

Algorithm 5 If $\mathcal{S}_{j}, j=1,2$, have the Fourier symbols

$$
\sigma_{j}=\mathcal{F}\left(\mathcal{S}_{j}\right)=\frac{1}{|\boldsymbol{k}|^{2}-2 \tilde{\omega}^{2}+2 i \tilde{\omega} \sigma Z+(2 i \tilde{\omega}+\sigma Z) s_{j}} M_{s_{j}}, \quad s_{j} \in \mathbb{C},
$$

then the Schwarz algorithm (3.2) has the convergence factor

$$
\rho_{5}\left(|\boldsymbol{k}|, \tilde{\omega}, \sigma, Z, L, s_{1}, s_{2}\right)=\left|\left(\frac{\sqrt{|\boldsymbol{k}|^{2}-\tilde{\omega}^{2}+i \tilde{\omega} \sigma Z}-i \tilde{\omega}}{\sqrt{|\boldsymbol{k}|^{2}-\tilde{\omega}^{2}+i \tilde{\omega} \sigma Z}+i \tilde{\omega}}\right)\right| \rho_{4}\left(|\boldsymbol{k}|, \tilde{\omega}, \sigma, Z, L, s_{1}, s_{2}\right) \text {. }
$$

The different symbols $\sigma_{j}$ depend on the choice of the parameters $s, s_{1}$ and $s_{2}$. In order to obtain an efficient algorithm, we will choose $\sigma_{j}, j=1,2$ such that $\rho_{l}$, $l=2, . ., 5$ is minimum over a range of frequencies. Therefore the parameters are solutions of the min-max problems

$\min _{s \in \mathbb{C}} \max _{|\boldsymbol{k}| \in K} \rho_{j}(|\boldsymbol{k}|, \tilde{\omega}, \sigma, Z, L, s), j=2,3, \quad \min _{s_{1}, s_{2} \in \mathbb{C}} \max _{|\boldsymbol{k}| \in K} \rho_{j}\left(|\boldsymbol{k}|, \tilde{\omega}, \sigma, Z, L, s_{1}, s_{2}\right), j=4,5$,

where $K$ denotes the set of relevant numerical frequencies. In the next section, we will analyze these min-max problems for each of the algorithms in Corollary 4.1. 
5. Optimized transmission conditions. In this section, we solve the various min-max problems seen in (4.12). The fundamental difference with the case $\sigma=0$ is that here we do not need to exclude the resonance frequencies (see [8]). This changes the nature of the min-max problems. We have more local maxima in $|\boldsymbol{k}|$, and balancing these maxima by the equioscillation principe, is more difficult. In a numerical implementation, the range of frequencies is bounded, i.e $|\boldsymbol{k}| \in K:=\left[k_{\min }, k_{\max }\right]$, where the minimum frequency $k_{\min }>0$ is a constant depending on the geometry, and the maximum numerical frequency that can be represented on a mesh is $k_{\max }=\frac{C}{h}$ where $C$ is a constant and $h$ is the mesh size.

Before solving the min-max problems in (4.12), we give an asymptotic expression for the maximum of the convergence factor (4.3) of the classical Schwarz algorithm over $|\boldsymbol{k}| \in K$. This allows us to see the behavior of the algorithm (3.1) when $h$ goes to zero.

COROLlary 5.1. The asymptotic convergence factor of the classical Schwarz method (Algorithm 1), for small mesh size $h$ is

$$
\max _{|\boldsymbol{k}| \in K} \rho_{1}(|\boldsymbol{k}|, \tilde{\omega}, \sigma, Z, L)= \begin{cases}1-\frac{4}{3}\left(9 \omega^{4} \sigma^{2} \mu^{3} \varepsilon C_{L}^{6}\right)^{\frac{1}{8}} h^{\frac{3}{4}}+O\left(h^{\frac{5}{4}}\right), & L=C_{L} h, \\ 1-\frac{\omega^{2} \sigma \sqrt{\mu^{3} \varepsilon}}{C^{3}} h^{3}+O\left(h^{5}\right), & L=0 .\end{cases}
$$

Proof. The proof is obtained by expanding the maximum of $\rho_{1}$ over $|\boldsymbol{k}| \in K$ for $h$ small.

5.1. Optimization of Algorithm 2. We look for $s$ under the form $s=p+i q$, with $(p, q) \in \mathbb{R}_{+}^{2}$ and $p=q$, such that $p$ is solution of the min-max problem

$$
\min _{p \geq 0}\left(\max _{|\boldsymbol{k}| \in K} \rho_{2}(|\boldsymbol{k}|, \tilde{\omega}, \sigma, Z, L, p(1+i))\right) .
$$

We choose $p=q$ in order to simplify the computations, this choice being justified for the Helmholtz equation case in [15]. The case where $p \neq q$ is discussed in [11].

The non-overlapping case. Using the change of variables

$$
\xi(|\boldsymbol{k}|):=\Re\left(\sqrt{|\boldsymbol{k}|^{2}-\tilde{\omega}^{2}+i \sigma \tilde{\omega} Z}\right), \quad y:=\sigma \tilde{\omega} Z,
$$

the convergence factor simplifies to

$$
\rho_{2}(\tilde{\omega}, Z, \sigma, 0,|\boldsymbol{k}|, p(1+i))=\sqrt{\frac{4 \xi^{2}(\xi-p)^{2}+(y-2 \xi p)^{2}}{4 \xi^{2}(\xi+p)^{2}+(y+2 \xi p)^{2}}}=: R(\xi, y, p) .
$$

Since the mapping $|\boldsymbol{k}| \mapsto \xi(|\boldsymbol{k}|)$ is increasing, the optimization problem (5.2) can be re-written as

$$
\min _{p \geq 0}\left(\max _{k_{\min } \leq|\boldsymbol{k}| \leq k_{\max }} \rho_{2}(\tilde{\omega}, Z, \sigma, 0,|\boldsymbol{k}|, p(1+i))\right)=\min _{p \geq 0}\left(\max _{\xi_{\min } \leq \xi \leq \xi_{\max }} R(\xi, y, p)\right),
$$

where $\xi_{\min }=\xi\left(k_{\min }\right)$ and $\xi_{\max }=\xi\left(k_{\max }\right)$. We can prove the following result 
THEOREM 5.2. In the non-overlapping case, the solution of the problem

$$
\min _{p \geq 0}\left(\max _{\xi_{\min } \leq \xi \leq \xi_{\max }} R(\xi, y, p)\right)
$$

is given by

$$
p^{*}= \begin{cases}\min \left(p_{2}, p_{23}\right), & \text { if } y<2 \xi_{\max } \xi_{\min }, \\ \min \left(p_{1}, p_{12}\right), & \text { if } y \geq 2 \xi_{\max } \xi_{\min },\end{cases}
$$

where $p_{1}, p_{2}, p_{12}$, and $p_{23}$ are constants defined by

$$
\begin{aligned}
& p_{12}=\frac{y^{\frac{1}{4}} \sqrt{\xi_{\min }\left(y+2 \xi_{\min }^{2}+\xi_{\min } \sqrt{2 y}\right)}}{2^{\frac{3}{4}} \xi_{\min }}, \quad p_{23}=\frac{y^{\frac{1}{4}} \sqrt{\xi_{\max }\left(y+2 \xi_{\max }^{2}+\xi_{\max } \sqrt{2 y}\right)}}{2^{\frac{3}{4}} \xi_{\max }}, \\
& p_{1}=\frac{\sqrt{8 \xi_{\min }^{4}+2 y^{2}}}{4 \xi_{\min }}, \quad p_{2}=\frac{\sqrt{8 \xi_{\max }^{4}+2 y^{2}}}{4 \xi_{\max }}
\end{aligned}
$$

Proof. We start by computing the partial derivative of $R$ with respect to $p$ in order to restrict the range of $p$ in the min-max problem,

$$
E_{1}:=\frac{\partial R}{\partial p}(\xi, y, p)=\frac{1}{R(\xi, y, p)} \frac{\left(32 \xi^{3} y+64 \xi^{5}\right)\left(p^{2}-\frac{1}{8} \frac{4 \xi^{4}+y^{2}}{\xi^{2}}\right)}{\left(4 \xi^{4}+8 \xi^{3} p+8 \xi^{2} p^{2}+y^{2}+4 y \xi p\right)^{2}}
$$

$E_{1}$ is negative for $p \in\left[0, \frac{\sqrt{8 \xi^{4}+2 y^{2}}}{4 \xi}\right]$ and positive for $p \in\left[\frac{\sqrt{8 \xi^{4}+2 y^{2}}}{4 \xi},+\infty\right)$. Moreover the minimum of the mapping $\xi \mapsto \frac{\sqrt{8 \xi^{4}+2 y^{2}}}{4 \xi}$ is $\sqrt{\frac{y}{2}}$. This implies that for $p \leq \sqrt{\frac{y}{2}}$, $R$ decreases with $p$ for all $\xi \in\left[\xi_{\text {min }},+\infty\right)$. Hence we can restrict the range of $p$ to the interval $\left[\sqrt{\frac{y}{2}},+\infty\right)$.

We now look for the eventual extrema of $\xi \mapsto R(\xi, y, p)$ by computing the zeros of the derivative of $R$ with respect to $\xi$,

$$
\frac{\partial R}{\partial \xi}(\xi, y, p)=\frac{4 p\left(\xi^{2}-\frac{1}{2} y\right)\left(8 \xi^{4}+16\left(y-p^{2}\right) \xi^{2}+2 y^{2}\right)}{R(\xi, y, p)\left(4 \xi^{4}+8 \xi^{3} p+8 \xi^{2} p^{2}+y^{2}+4 y \xi p\right)^{2}} .
$$

The polynomial $P(\xi)=\left(\xi^{2}-\frac{1}{2} y\right)\left(8 \xi^{4}+16\left(y-p^{2}\right) \xi^{2}+2 y^{2}\right)$ has at most three positive roots, and $\xi_{2}=\sqrt{\frac{y}{2}}$ is always a root. The other roots are given by

$$
\xi_{1,3}=\frac{1}{2} \sqrt{-4 y+4 p^{2} \mp 2 \sqrt{\left(3 y-2 p^{2}\right)\left(y-2 p^{2}\right)}} .
$$

We now show that for $p \geq \sqrt{3 y / 2}, \xi_{2}$ is a maximum and the other two roots of $P$ cannot be maxima. The second partial derivative of $R$ with respect to $\xi$ at $\xi_{2}$ is

$$
\frac{\partial^{2} R}{\partial \xi^{2}}\left(\xi_{2}, y, p\right)=-4 \sqrt{\frac{2}{y}} \frac{\left(2 p^{2}-3 y\right) p}{|\sqrt{2} p-\sqrt{y}|(\sqrt{2} p+\sqrt{y})^{3}},
$$

which shows that $\xi_{2}$ is a local maximum if $p<\sqrt{3 y / 2}$. Moreover in this case, the other two roots are real and these are minima because $\xi_{1} \leq \xi_{2} \leq \xi_{3}$. In the case $p \geq \sqrt{3 y / 2}, \xi_{2}$ is a local minimum and the other two roots are not real. Therefore the maximum of $R$ is either at $\xi_{\min }, \xi_{2}$ or $\xi_{\max }$.

From the expression (5.4) we can derive the following properties of $R$ : 
(a) The mapping $p \mapsto R\left(\xi_{\min }, y, p\right)$ is decreasing on $\left[\sqrt{\frac{y}{2}}, p_{1}\right]$ and increasing on $\left[p_{1},+\infty\right)$.

(b) The mapping $p \mapsto R\left(\xi_{2}, y, p\right)$ is increasing on $\left[\sqrt{\frac{y}{2}},+\infty\right)$.

(c) The mapping $p \mapsto R\left(\xi_{\max }, y, p\right)$ is decreasing on $\left[\sqrt{\frac{y}{2}}, p_{2}\right]$ and increasing on $\left[p_{2},+\infty\right)$.

To conclude the proof of the theorem, we need the following lemmas:

LEMMA 5.3. Let $p_{13}$ be the constant defined by

$$
p_{13}=\frac{\sqrt{2 \xi_{\min } \xi_{\max }\left(y^{2}+2 y \xi_{\min }^{2}+4 y \xi_{\min } \xi_{\max }+2 y \xi_{\max }^{2}+4 \xi_{\min }^{2} \xi_{\max }^{2}\right)}}{4 \xi_{\min } \xi_{\max }} .
$$

Then the following properties are verified:

i) $p_{12}$ is the unique solution of the equation $R\left(\xi_{\min }, y, p\right)=R\left(\xi_{2}, y, p\right)$ on $\mathbb{R}_{+} \backslash\{0\}$.

ii) $p_{13}$ is the unique solution of the equation $R\left(\xi_{\min }, y, p\right)=R\left(\xi_{\max }, y, p\right)$ on $\mathbb{R}_{+} \backslash\{0\}$.

iii) $p_{23}$ is the unique solution of the equation $R\left(\xi_{2}, y, p\right)=R\left(\xi_{\max }, y, p\right)$ on $\mathbb{R}_{+} \backslash\{0\}$.

iv) We have

$$
\max \left(p_{1}, p_{2}\right)= \begin{cases}p_{2}, & \text { if } y<2 \xi_{\max } \xi_{\min } \\ p_{1}, & \text { if } y \geq 2 \xi_{\max } \xi_{\min }\end{cases}
$$

v) We have

$$
\max \left(p_{12}, p_{23}\right)= \begin{cases}p_{23}, & \text { if } y<2 \xi_{\max } \xi_{\min } \\ p_{12}, & \text { if } y \geq 2 \xi_{\max } \xi_{\min }\end{cases}
$$

vi) We have $\sqrt{\frac{3 y}{2}} \leq p_{12}, p_{23} \leq p_{13}$.

vii) We have

$$
\max _{\xi_{\min } \leq \xi \leq \xi_{\max }} R(\xi, y, p)= \begin{cases}R\left(\xi_{\max }, y, p\right), & \text { if } y<2 \xi_{\max } \xi_{\min } \text { and } p \in\left[\sqrt{\frac{y}{2}}, p_{23}\right], \\ R\left(\xi_{2}, y, p\right), & \text { if } y<2 \xi_{\max } \xi_{\min } \text { and } p \in\left[p_{23},+\infty\right), \\ R\left(\xi_{\min }, y, p\right), & \text { if } y \geq 2 \xi_{\max } \xi_{\min } \text { and } p \in\left[\sqrt{\frac{y}{2}}, p_{12}\right], \\ R\left(\xi_{2}, y, p\right), & \text { if } y \geq 2 \xi_{\max } \xi_{\min } \text { and } p \in\left[p_{12},+\infty\right)\end{cases}
$$

Proof. To prove the first three properties, we compute

$$
\begin{aligned}
R\left(\xi_{b}, y, p\right)^{2} & -R\left(\xi_{m}, y, p\right)^{2} \\
& =\frac{8 p\left(\xi_{m}-\xi_{b}\right)\left(2 \xi_{m} \xi_{b}-y\right)\left(8 \xi_{b} \xi_{m} p^{2}-4 \xi_{b}^{2} \xi_{m}^{2}-2 \xi_{b}^{2} y-4 y \xi_{m} \xi_{b}-2 y \xi_{m}^{2}-y^{2}\right)}{\left(4 \xi_{b}^{2}\left(\xi_{b}+p\right)^{2}+\left(y+2 \xi_{b} p\right)^{2}\right)\left(4 \xi_{m}^{2}\left(\xi_{m}+p\right)^{2}+\left(y+2 \xi_{m} p\right)^{2}\right)} .
\end{aligned}
$$

This quantity is zero if

$$
p=\frac{\sqrt{2 \xi_{b} \xi_{m}\left(y^{2}+2 y \xi_{b}^{2}+4 y \xi_{b} \xi_{m}+2 y \xi_{m}^{2}+4 \xi_{b}^{2} \xi_{m}^{2}\right)}}{4 \xi_{b} \xi_{m}} .
$$

By replacing successively the pairs $\left\{\xi_{b}, \xi_{m}\right\}$ by $\left\{\xi_{\min }, \xi_{2}\right\},\left\{\xi_{\min }, \xi_{\max }\right\}$ and $\left\{\xi_{2}, \xi_{\max }\right\}$ in (5.6) we get the desired result.

To prove property $i v$ ), we compute

$$
p_{1}^{2}-p_{2}^{2}=\frac{\left(\xi_{\max }-\xi_{\min }\right)\left(\xi_{\min }+\xi_{\max }\right)\left(y-2 \xi_{\max } \xi_{\min }\right)\left(y+2 \xi_{\max } \xi_{\min }\right)}{8 \xi_{\max }^{2} \xi_{\min }^{2}}
$$


which shows that $p_{1} \leq p_{2}$ if $y<2 \xi_{\max } \xi_{\min }$ and $p_{1} \geq p_{2}$ if $y \geq 2 \xi_{\max } \xi_{\min }$.

To prove property $i v)$, we compute

$$
p_{12}^{2}-p_{23}^{2}=-\frac{\sqrt{y} \sqrt{2}\left(\xi_{\max }-\xi_{\min }\right)\left(2 \xi_{\max } \xi_{\min }-y\right)}{4 \xi_{\max } \xi_{\min }} .
$$

We can see that $p_{12} \leq p_{23}$ if $y<2 \xi_{\max } \xi_{\min }$ and $p_{12} \geq p_{23}$ if $y \geq 2 \xi_{\max } \xi_{\min }$.

We now show vi) by computing

$$
\begin{aligned}
& p_{12}^{2}-p_{13}^{2}=-\frac{\left(2 y+4 \xi_{\min }^{2}\right)\left(\xi_{\max }-1 / 2 \sqrt{y} \sqrt{2}\right)^{2}}{8 \xi_{\max } \xi_{\min }} \leq 0, \quad p_{23}^{2}-p_{13}^{2}=-\frac{\left(4 \xi_{\max }^{2}+2 y\right)\left(\xi_{\min }-1 / 2 \sqrt{y} \sqrt{2}\right)^{2}}{8 \xi_{\max } \xi_{\min }} \leq 0 \\
& p_{12}^{2}-\frac{3 y}{2}=\frac{\sqrt{y} \sqrt{2}\left(\xi_{\min }-1 / 2 \sqrt{y} \sqrt{2}\right)^{2}}{2 \xi_{\min }} \geq 0, \quad p_{23}^{2}-\frac{3 y}{2}=\frac{\sqrt{y} \sqrt{2}\left(\xi_{\max }-1 / 2 \sqrt{y} \sqrt{2}\right)^{2}}{2 \xi_{\max }} \geq 0
\end{aligned}
$$

This shows that $p_{i 2}^{2}-\frac{3 y}{2} \geq 0$ and $p_{i 2}^{2}-p_{13}^{2} \leq 0, i=1,2$.

Finally we treat the last point. After some computations we obtain

$$
\begin{aligned}
E_{2} & :=R\left(\xi_{\min }, y, p\right)^{2}-R\left(\xi_{\max }, y, p\right)^{2} \\
& =\frac{64 p \xi_{\min } \xi_{\max }\left(\xi_{\max }-\xi_{\min }\right)\left(2 \xi_{\max } \xi_{\min }-y\right)\left(p^{2}-p_{13}{ }^{2}\right)}{\left(4 \xi_{\min }^{2}\left(\xi_{\min }+p\right)^{2}+\left(y+2 \xi_{\min } p\right)^{2}\right)\left(4 \xi_{\max }^{2}\left(\xi_{\max }+p\right)^{2}+\left(y+2 \xi_{\max } p\right)^{2}\right)} \\
E_{3} & :=R\left(\xi_{\max }, y, p\right)^{2}-R\left(\xi_{2}, y, p\right)^{2} \\
& =-\frac{32 p \xi_{\max }\left(\xi_{\max }-1 / 2 \sqrt{y} \sqrt{2}\right)^{2}\left(p^{2}-p_{23}^{2}\right)}{\left(4 \xi^{2}(\xi+p)^{2}+(y+2 \xi p)^{2}\right)\left(y+2 \sqrt{y} \sqrt{2} p+2 p^{2}\right)} \\
E_{4} & :=R\left(\xi_{\min }, y, p\right)^{2}-R\left(\xi_{2}, y, p\right)^{2} \\
& =-\frac{32 p \xi_{\min }\left(\xi_{\min }-1 / 2 \sqrt{y} \sqrt{2}\right)^{2}\left(p^{2}-p_{12}^{2}\right)}{\left(4 \xi^{2}(\xi+p)^{2}+(y+2 \xi p)^{2}\right)\left(y+2 \sqrt{y} \sqrt{2} p+2 p^{2}\right)}
\end{aligned}
$$

From the expression above we can easily see that

- if $y<2 \xi_{\max } \xi_{\min }$ and $p \in\left[\sqrt{\frac{\bar{y}}{2}}, p_{23}\right]$, then $E_{2} \leq 0$ because $p_{13} \geq p_{23}$ (see proposition (vi)) and $E_{3} \geq 0$.

- if $y<2 \xi_{\max } \xi_{\min }$ and $p \in\left[p_{23},+\infty\right)$, then $E_{4} \leq 0$ since $p_{12} \leq p_{23}$ (see proposition (vi)) and $E_{3} \leq 0$.

- If $y \geq 2 \xi_{\max } \xi_{\min }$ and $p \in\left[\sqrt{\frac{y}{2}}, p_{12}\right]$, then $E_{2} \geq 0$ and $E_{4} \geq 0$.

- If $y \geq 2 \xi_{\max } \xi_{\min }$ and $p \in\left[p_{12},+\infty\right)$, then $E_{3} \leq 0$ since $p_{12} \leq p_{23}$ (see proposition (vi)) and $E_{4} \leq 0$.

This Lemma proves that the solution of the min-max problem (5.2) depends mainly on the sign of $y-2 \xi_{\max } \xi_{\min }$. We can see on Figure 5.1 the solution of the min-max problem for different values of $y, \xi_{\min }$ and $\xi_{\max }$. The pictures (a), (b), (c) and (d) show the four cases of the theorem $\left(y<2 \xi_{\max } \xi_{\min }, p_{2} \geq p_{23}\right),(y \geq$ $\left.2 \xi_{\max } \xi_{\min }, p_{1} \geq p_{12}\right),\left(y<2 \xi_{\max } \xi_{\min }, p_{2} \leq p_{23}\right)$ and $\left(y \geq 2 \xi_{\max } \xi_{\min }, p_{1} \leq p_{12}\right)$.

LEMMA 5.4. If $y<2 \xi_{\max } \xi_{\min }$, the solution of the min-max problem

$$
\min _{\sqrt{\frac{y}{2}} \leq p \leq p_{23}}\left(\max _{\xi_{\min } \leq \xi \leq \xi_{\max }} R(\xi, y, p)\right)
$$

is $p^{*}=\min \left(p_{2}, p_{23}\right)$ and the solution of the min-max problem

$$
\min _{p \geq p_{23}}\left(\max _{\xi_{\min } \leq \xi \leq \xi_{\max }} R(\xi, y, p)\right)
$$


(a)

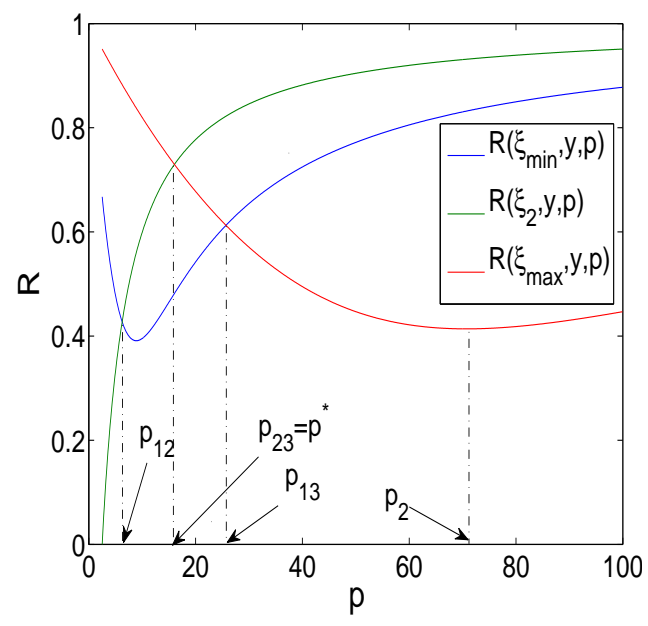

(c)



(b)



(d)

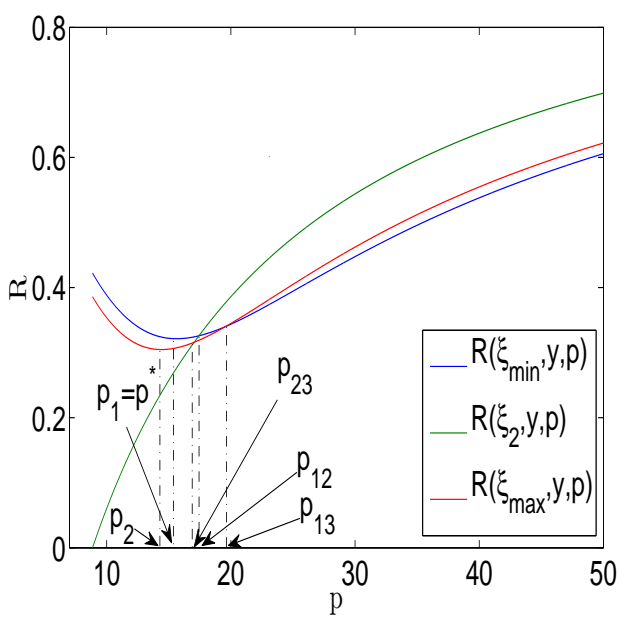

FiG. 5.1. Solutions of the min-max problem for different values of $y, \xi_{\min }$ and $\xi_{\max }$. (a) : $y=12.5, \xi_{\min }=0.5, \xi_{\max }=100,(b): y=125.5, \xi_{\min }=0.5, \xi_{\max }=100,(c): y=157$, $\xi_{\min }=3.5, \xi_{\max }=24$, (d) :y=157, $\xi_{\min }=3.6, \xi_{\max }=20$.

is $p^{*}=p_{23}$.

Proof. By using the property (vii) of Lemma 5.3, for $p \in\left[\sqrt{\frac{y}{2}}, p_{23}\right]$, we have

$$
R\left(\xi_{\max }, y, p\right)=\max _{\xi_{\min } \leq \xi \leq \xi_{\max }} R(\xi, y, p) .
$$

Hence the solution of the first min-max problem is the minimum of the mapping $p \mapsto R\left(\xi_{\max }, y, p\right)$ on $\left[\sqrt{\frac{y}{2}}, p_{23}\right]$. By the property $(c)$ of page 7 , the minimum of $R\left(\xi_{\max }, y, \cdot\right)$ on $\left[\sqrt{\frac{y}{2}}, p_{23}\right]$ is $\min \left(p_{2}, p_{23}\right)$.

By using the property (vii) of Lemma 5.3 , for $p \in\left[p_{23},+\infty\right.$ ), we have

$$
R\left(\xi_{2}, y, p\right)=\max _{\xi_{\min } \leq \xi \leq \xi_{\max }} R(\xi, y, p) .
$$


Therefore the solution of the second min-max problem is the minimum of the mapping $R\left(\xi_{2}, y, \cdot\right)$ on $\left[p_{23},+\infty\right)$. By property $(b)$ of page 7 , the minimum of $R\left(\xi_{2}, y, \cdot\right)$ on $\left[p_{23},+\infty\right)$ is $p_{23}$.

LEMMA 5.5. If $y \geq 2 \xi_{\max } \xi_{\min }$, the solution of the min-max problem

$$
\min _{\sqrt{\frac{y}{2} \leq p \leq p_{12}}}\left(\max _{\xi_{\min } \leq \xi \leq \xi_{\max }} R(\xi, y, p)\right)
$$

is $p^{*}=\min \left(p_{1}, p_{12}\right)$ and the solution of the min-max problem

$$
\min _{p \geq p_{12}}\left(\max _{\xi_{\min } \leq \xi \leq \xi_{\max }} R(\xi, y, p)\right)
$$

is $p^{*}=p_{12}$.

Proof. By using the property (vii) of Lemma 5.3, for $p \in\left[\sqrt{\frac{y}{2}}, p_{12}\right]$, we have

$$
R\left(\xi_{\min }, y, p\right)=\max _{\xi_{\min } \leq \xi \leq \xi_{\max }} R(\xi, y, p) .
$$

The solution of the first min-max problem is the minimum of the mapping $p \mapsto$ $R\left(\xi_{\min }, y, p\right)$ on $\left[\sqrt{\frac{y}{2}}, p_{12}\right]$. Using now $(a)$ of page 7 , the minimum of $R\left(\xi_{\min }, y, \cdot\right)$ on $\left[\sqrt{\frac{y}{2}}, p_{12}\right]$ is $\min \left(p_{1}, p_{12}\right)$.

By proposition (vii) of Lemma 5.3, for $p \in\left[p_{12},+\infty\right.$ ), we have

$$
R\left(\xi_{2}, y, p\right)=\max _{\xi_{\min } \leq \xi \leq \xi_{\max }} R(\xi, y, p) .
$$

Therefore the solution of the second min-max problem is the minimum of the mapping $p \mapsto R\left(\xi_{2}, y, p\right)$ on $\left[p_{12},+\infty\right)$. By property $(a)$ on page 7 , the minimum of $R\left(\xi_{2}, y, \cdot\right)$ on $\left[p_{23},+\infty\right)$ is $p_{12}$.

We can now return to the end of the theorem. The min-max problem (5.2) can be rewritten in the equivalent form

$$
\begin{array}{r}
\min _{p \geq \sqrt{\frac{y}{2}}}\left(\max _{\xi_{\min } \leq \xi \leq \xi_{\max }} R(\xi, y, p)\right)=\min \left(\min _{\sqrt{\frac{y}{2}} \leq p \leq p_{23}}\left(\max _{\xi_{\min } \leq \xi \leq \xi_{\max }} R(\xi, y, p)\right),\right. \\
\left.\min _{p \geq p_{23}}\left(\max _{\xi_{\min } \leq \xi \leq \xi \max } R(\xi, y, p)\right)\right)
\end{array}
$$

which is useful for the case $y<2 \xi_{\max } \xi_{\min }$, because we then get, using Lemma 5.4

$$
\min _{p \geq \sqrt{\frac{y}{2}}}\left(\max _{\xi_{\min } \leq \xi \leq \xi_{\max }} R(\xi, y, p)\right)=R\left(\xi_{\max }, y, \min \left(p_{2}, p_{23}\right)\right) .
$$

The min-max problem (5.2) can also be rewritten as

$$
\begin{aligned}
& \min _{p \geq \sqrt{\frac{y}{2}}}\left(\max _{\xi_{\min } \leq \xi \leq \xi_{\max }} R(\xi, y, p)\right)=\min \left(\min \sqrt{\frac{y}{2} \leq p \leq p_{12}}\left(\max _{\xi_{\min } \leq \xi \leq \xi_{\max }} R(\xi, y, p)\right),\right. \\
& \left.\min _{p \geq p_{12}}\left(\max _{\xi_{\min } \leq \xi \leq \xi_{\max }} R(\xi, y, p)\right)\right) \text {, }
\end{aligned}
$$


which is now usefull for the case $y \geq 2 \xi_{\max } \xi_{\min }$, because we then get, using Lemma 5.5 ,

$$
\min _{p \geq \sqrt{\frac{y}{2}}}\left(\max _{\xi_{\min } \leq \xi \leq \xi_{\max }} R(\xi, y, p)\right)=R\left(\xi_{\max }, y, \min \left(p_{1}, p_{12}\right)\right)
$$

COROLlary 5.6. For $h$ sufficiently small in the non-overlapping case, $L=0$, the solution of the min-max problem (5.2) is given by

$$
p^{*}=\frac{(\omega \sigma \mu)^{\frac{1}{4}} \sqrt{C}}{2^{\frac{1}{4}} \sqrt{h}} \quad \text { and } \quad \rho_{2}^{*}=1-\frac{2^{\frac{3}{4}}(\omega \sigma \mu)^{\frac{1}{4}} \sqrt{h}}{\sqrt{C}}+O(h) .
$$

Proof. If we assume $h$ sufficiently small, then $\xi_{\max }=\xi(C / h)$ is large. By this assumption, the solution of the min-max problem (5.2) is given by the first equation of (5.3), i.e

$$
p^{*}=\frac{\sqrt[4]{2 y} \sqrt{\xi_{\max }\left(y+2 \xi_{\max }^{2}+\xi_{\max } \sqrt{2} \sqrt{y}\right)}}{2 \xi_{\max }} .
$$

By expanding $p^{*}$ for $h$ small, using that $\xi_{\max }=\xi(C / h)$, we get the desired result. Corollary 5.6 gives the optimal parameter $p^{*}$ and the corresponding convergence factor of algorithm 2 in terms of the mesh size $h$. In some practical situations, where we consider high frequencies, it is interesting to give the optimized parameters in term of $\omega$. For the high frequencies, it has been shown in [2] that it is necessary, to avoid the pollution effect, to couple the frequency $\omega$ with $h$ by the relation $h=C_{h} / \omega^{\gamma}$; where $\gamma \geq 1$ depends on the discretization method. In [2], it has been shown that $\gamma=\frac{3}{2}$ for a $P_{1}$ finite element method. We have the following corollary:

COROLlary 5.7. Let $h=C_{h} / \omega^{\gamma}$. If $\gamma=1$, then for $\omega$ large, the solution of the min-max problem (5.2) is given by

$$
p^{*}=\frac{(\sigma \mu)^{\frac{1}{4}}\left(C^{2}-\varepsilon \mu C_{h}^{2}\right)^{\frac{1}{4}}}{2^{\frac{1}{4}} \sqrt{C_{h}}} \omega^{\frac{3}{4}} \quad \text { and } \quad \rho_{2}^{*}=1-\frac{2^{\frac{3}{4}}(\sigma \mu)^{\frac{1}{2}} \sqrt{C_{h}}}{\left(C^{2}-\varepsilon \mu C_{h}^{2}\right)^{\frac{1}{4}}} \omega^{-\frac{1}{4}} .
$$

If $\gamma>1$, for $\omega$ large, the solution is

$$
p^{*}=\frac{(\sigma \mu)^{\frac{1}{4}} \sqrt{C}}{2^{\frac{1}{4}} \sqrt{C_{h}}} \omega^{\frac{2 \gamma+1}{4}} \quad \text { and } \quad \rho_{2}^{*}=1-\frac{2^{\frac{3}{4}}(\sigma \mu)^{\frac{1}{4}} \sqrt{C_{h}}}{\sqrt{C}} \omega^{\frac{1-2 \gamma}{4}} .
$$

Proof. If we assume $\omega$ sufficiently large, then $\xi_{\max }=\xi\left(\omega C / C_{h}\right)$ is large. By this assumption, the solution of the min-max problem (5.2) is given by the first equation of (5.3), i.e

$$
p^{*}=\frac{\sqrt[4]{2 y} \sqrt{\xi_{\max }\left(y+2 \xi_{\max }^{2}+\xi_{\max } \sqrt{2} \sqrt{y}\right)}}{2 \xi_{\max }} .
$$

By expanding $p^{*}$ for $\omega$ large, using that $\xi_{\max }=\xi\left(\omega C / C_{h}\right)$, we get the desired result. 
The overlapping case. After the complete analysis of the best approximation problem without overlap in the previous paragraph, we focus in the remainder of this paper on asymptotic analysis, in order to be able to present a complete set of optimized transmission conditions for all algorithms. We start with the case of the previous paragraph, but now consider an overlapping method. We set $L=h, k_{\min }=0$, $k_{\max }=\frac{\pi}{h}$ and denote by $p^{*}$ the solution of the min-max problem (5.2). Solving this min-max problem numerically for different parameter values and different mesh sizes $h$, we observe that the solution of (5.2) equioscillates once, i.e. $p^{*}$ is solution of

$$
\rho_{2}\left(\bar{k}_{1}, \tilde{\omega}, \sigma, Z, L, p^{*}(1+i)\right)=\rho_{2}\left(\bar{k}_{2}, \tilde{\omega}, \sigma, Z, L, p^{*}(1+i)\right),
$$

where $\bar{k}_{1}$ and $\bar{k}_{2}$ are two interior local maxima of $\rho_{2}$. For small $h$, we see numerically that these maxima and the optimized parameter $p^{*}$ behave like

$$
\bar{k}_{1}=C_{b_{1}}, \bar{k}_{2}=C_{b_{2}} h^{-\frac{2}{3}} \text {, and } p^{*}=C_{p} h^{-\frac{1}{3}} \text {, for some constants } C_{p}, C_{b_{1}}, C_{b_{2}} .
$$

In order to determine the constants $C_{b_{1}}, C_{b_{2}}$ and $C_{p}$, we solve now the corresponding equations asymptotically: since $\bar{k}_{1}$ and $\bar{k}_{2}$ are local maxima of the convergence factor, the corresponding derivatives must vanish, and we find asymptotically

$$
\begin{aligned}
& \frac{\partial \rho_{2}}{\partial k}\left(\overline{k_{1}}, \tilde{\omega}, \sigma, Z, L, p^{*}(1+i)\right)=0 \Rightarrow C_{b_{1}}=\tilde{\omega} \\
& \frac{\partial \rho_{2}}{\partial k}\left(\overline{k_{2}}, \tilde{\omega}, \sigma, Z, L, p^{*}(1+i)\right)=0 \Rightarrow C_{b_{2}}=\sqrt{2 C_{p}} .
\end{aligned}
$$

The equioscillation equation (5.10) must also be satisfied, which gives asymptotically

$$
\rho_{2}\left(\overline{k_{1}}, \tilde{\omega}, \sigma, Z, L, p^{*}(1+i)\right)=\rho_{2}\left(\overline{k_{2}}, \tilde{\omega}, \sigma, Z, L, p^{*}(1+i)\right) \Rightarrow \frac{\sqrt{2} \sqrt{\tilde{\omega} \sigma Z}}{C_{p}}=\frac{C_{b_{2}}{ }^{2}+2 C_{p}}{C_{b_{2}}} .
$$

Solving the three equations obtained for the constants $C_{b_{1}}, C_{b_{2}}$ and $C_{p}$, and expressing the results in the original problem parameters $\omega, \epsilon, \mu$ and $\sigma$, we find the asymptotic result

$$
p^{*}=\frac{(2 \omega \sigma \mu)^{\frac{1}{3}}}{2 h^{\frac{1}{3}}}, \quad \rho_{2}^{*}=1-2^{\frac{7}{6}}(\omega \sigma \mu)^{\frac{1}{6}} h^{\frac{1}{3}} .
$$

Lemma 5.8. Asymptotically, the parameter $p^{*}$ defined in (5.11) is a local minimum of the min-max problem (5.2).

Proof. The parmeter $p^{*}$ is a local minimum, if there exists no variation $\delta p$ such that $\rho_{2}\left(k, \tilde{\omega}, \sigma, Z, 0,\left(p^{*}+\delta p\right)(1+i)\right) \leq \rho_{2}\left(k, \tilde{\omega}, \sigma, Z, 0, p^{*}(1+i)\right)$, for $k=\bar{k}_{1}, \bar{k}_{2}$. By the Taylor formula, it suffices to prove that there is no variation $\delta p$ such that $\delta p \frac{\partial \rho_{2}}{\partial p}\left(k, \tilde{\omega}, \sigma, Z, 0, p^{*}(1+i)\right)<0$, for $k=\bar{k}_{1}, \bar{k}_{2}$. To prove this, it is necessary to obtain the next higher order terms in the expansions of $p^{*}, \bar{k}_{1}$ and $\bar{k}_{2}$. After a lengthy computation, we find that

$$
\bar{k}_{1} \sim \tilde{\omega}+\tilde{C}_{b_{1}} h^{\frac{2}{3}}, \quad \bar{k}_{2} \sim C_{b_{2}} h^{-\frac{2}{3}}+\tilde{C}_{b_{2}}, \quad p^{*} \sim C_{p} h^{-\frac{1}{3}}+\tilde{C}_{p} h^{\frac{1}{3}} .
$$

With these new three constants determined, we obtain for the derivative of $\rho_{3}$

$$
\frac{\partial \rho_{2}}{\partial p}\left(k_{1}, \tilde{\omega}, \sigma, Z, h, p^{*}(1+i)\right) \sim 4 \frac{2^{\frac{5}{6}} h^{\frac{2}{3}}}{(\omega \sigma \mu)^{\frac{1}{6}}}, \frac{\partial \rho_{2}}{\partial p}\left(\bar{k}_{2}, \tilde{\omega}, \sigma, Z, h, p^{*}(1+i)\right) \sim-2 \frac{2^{\frac{5}{6}} h^{\frac{2}{3}}}{(\omega \sigma \mu)^{\frac{1}{6}}} .
$$

Since these leading terms differ in sign, there can not be a variation $\delta p$ which diminishes the contraction factor obtained for $p^{*}$, and we have asymptotically a local minimum. $\square$

In Figure 5.2 we can see the convergence factor in the non-overlapping and overlapping case for the algorithm 2 compared to the classical one. 
5.2. Optimization of algorithm 3. Here, we look for $s$ of the form $s=p(1+i)$, such that $p$ is solution of the min-max problem

$$
\min _{p \geq 0}\left(\max _{k \in K} \rho_{3}(k, \tilde{\omega}, \sigma, Z, L, p(1+i))\right) .
$$

Again we are only searching for an asymptotic solution of this best approximation problem.

The non-overlapping case. Setting $L=0, k_{\min }=0, k_{\max }=\frac{C}{h}$ and denoting by $p^{*}$ the solution of $(5.12)$, we obtain from numerical experiments that the solution of (5.12) equioscillates once, i.e. $p^{*}$ is solution of

$$
\rho_{3}\left(k_{\max }, \tilde{\omega}, \sigma, Z, 0, p^{*}(1+i)\right)=\rho_{3}\left(\bar{k}, \tilde{\omega}, \sigma, Z, 0, p^{*}(1+i)\right),
$$

where $k_{b}$ is an interior local maximun of $\rho_{3}$. For small $h$ we find the asymptotic behavior $\bar{k}=C_{b} h^{-\frac{1}{7}}, p^{*}=C_{p} h^{-\frac{4}{7}}$, and to determine $C_{b}$ and $C_{p}$, we solve asymptotically

$$
\frac{\partial \rho_{3}}{\partial k}\left(\bar{k}, \tilde{\omega}, \sigma, Z, 0, p^{*}(1+i)\right)=0 \Rightarrow C_{p}=\frac{C_{b}^{4}}{3 \tilde{\omega}^{2} \sigma Z},
$$

and the equioscillation equation (5.13), which leads to $C_{b}=\left(6 C \tilde{\omega}^{4} \sigma^{2} Z^{2}\right)^{\frac{1}{7}}$. Solving the two equations for the two constants, and using again the original parameters $\omega$, $\epsilon, \mu$ and $\sigma$, we find

$$
p^{*}=\frac{1}{3} 6^{\frac{4}{7}} C^{\frac{4}{7}} \omega^{\frac{2}{7}} \mu^{\frac{3}{14}} \epsilon^{\frac{1}{14}} \sigma^{\frac{1}{7}} h^{-\frac{4}{7}}, \rho_{3}^{*}=1-\frac{4}{3} \frac{6^{\frac{4}{7}} \omega^{\frac{2}{7}} \mu^{\frac{3}{14}} \epsilon^{\frac{1}{14}} \sigma^{\frac{1}{7}} h^{\frac{3}{7}}}{C^{\frac{3}{7}}} .
$$

Lemma 5.9. Asymptotically, the parameter $p^{*}$ defined in (5.14) is a local minimum of the min-max problem (5.12).

Proof. The proof follows the same argument as the proof of Lemma 5.8. We obtain after a lengthy computation that

$$
\bar{k} \sim C_{b} h^{-\frac{1}{7}}+C_{b_{2}} h^{\frac{1}{7}}, \quad p^{*} \sim C_{p} h^{-\frac{4}{7}}+C_{p_{2}} h^{-\frac{2}{7}} .
$$

Determining the constants in the higher order terms as well, we can show that

$\frac{\partial \rho_{3}}{\partial p}\left(k_{\max }, \tilde{\omega}, \sigma, Z, 0, p^{*}(1+i)\right) \sim-\frac{4 h}{C}, \frac{\partial \rho_{3}}{\partial p}\left(\bar{k}, \tilde{\omega}, \sigma, Z, 0, p^{*}(1+i)\right) \sim \frac{1}{4} \frac{2^{\frac{5}{7}} 3^{\frac{5}{7}} \sigma^{\frac{3}{7}} Z^{\frac{3}{7}} h^{\frac{9}{7}}}{\tilde{\omega}^{\frac{1}{7}} C^{\frac{9}{7}}}$,

which shows by the opposite sign that $p^{*}$ is indeed a local minimum.

The overlapping case. Also with $L=h$ the solution of the min-max problem (5.12) equioscillates once, i.e. $p^{*}$ is solution of

$$
\rho_{3}\left(\bar{k}_{1}, \tilde{\omega}, \sigma, Z, L, p^{*}(1+i)\right)=\rho_{3}\left(\bar{k}_{2}, \tilde{\omega}, \sigma, Z, L, p^{*}(1+i)\right),
$$

where $\bar{k}_{1}$ and $\bar{k}_{2}$ are two interior local maxima of $\rho_{3}$. For small $h$, we get

$$
\bar{k}_{1}=C_{b_{1}} h^{-\frac{1}{10}}, \quad \bar{k}_{2}=C_{b_{2}} h^{-\frac{7}{10}}, \quad p^{*}=C_{p} h^{-\frac{2}{5}},
$$

and solving the corresponding equations asymptotically, we find in the original parameters $\omega, \epsilon, \mu$ and $\sigma$

$$
p^{*}=\frac{1}{3} \frac{3^{\frac{2}{5}} \varepsilon^{\frac{1}{10}} \mu^{\frac{3}{10}} \omega^{\frac{2}{5}} \sigma^{\frac{1}{5}} 2^{\frac{2}{5}}}{h^{\frac{2}{5}}}, \quad \rho_{3}^{*}=1-\frac{2}{3} 3^{\frac{7}{10}} 2^{\frac{7}{10}} \omega^{\frac{1}{5}} \varepsilon^{\frac{1}{20}} \mu^{\frac{3}{20}} \sigma^{\frac{1}{10}} h^{\frac{3}{10}}
$$

In Figure 5.2 we can see the convergence factor in the non-overlapping and overlapping case for algorithm 3 compared to the classical one and algorithm 2 . 
5.3. Optimization of algorithm 4. Here, we look for $s_{1}$ and $s_{2}$ of the form $s_{l}=p_{l}(1+i), l=1,2$ such that the $p_{l}$ are solution of the min-max problem

$$
\min _{p_{1}, p_{2} \geq 0}\left(\max _{k \in K} \rho_{4}\left(k, \tilde{\omega}, \sigma, Z, L, p_{1}(1+i), p_{2}(1+i)\right)\right) .
$$

As for algorithm 3, we proceed directly with the asymptotic analysis.

The non-overlapping case. Letting $L=0, k_{\min }=0, k_{\max }=\frac{C}{h}$ and denoting by $\left(p_{1}^{*}, p_{2}^{*}\right)$ the solution of the min-max problem (5.16), we find numerically that the solution of (5.16) equioscillates twice, i.e. $\left(p_{1}^{*}, p_{2}^{*}\right)$ is solution of

$$
\begin{aligned}
\rho_{4}\left(\bar{k}_{1}, \tilde{\omega}, \sigma, Z, L, p_{1}^{*}(1+i), p_{2}^{*}(1+i)\right) & =\rho_{4}\left(\bar{k}_{2}, \tilde{\omega}, \sigma, Z, L, p_{1}^{*}(1+i), p_{2}^{*}(1+i)\right) \\
& =\rho_{4}\left(k_{\max }, \tilde{\omega}, \sigma, Z, L, p_{1}^{*}(1+i), p_{2}^{*}(1+i)\right),
\end{aligned}
$$

where $\bar{k}_{1}$ and $\bar{k}_{2}$ are two interior local maxima of $\rho_{4}$. One can to show that $\bar{k}_{1}=\tilde{\omega}$, and for small $h$ we get

$$
\bar{k}_{2}=C_{b_{2}} h^{-\frac{1}{2}}, \quad p_{1}^{*}=C_{p} h^{-\frac{3}{4}}, \quad p_{2}^{*}=C_{q} h^{-\frac{3}{4}} .
$$

To determine the constants $C_{b_{2}}, C_{p}$ and $C_{q}$, we solve asymptotically the equioscillation conditions and the equation stating that $\bar{k}_{2}$ is a maximum, which leads to the system

$$
C_{b 2}=\sqrt{2 C_{p} C_{q}}, \quad C_{q}=\frac{1}{2} \frac{C_{p}^{3}}{C^{2}}, \quad C_{p}=(2 \tilde{\omega} \sigma Z)^{\frac{1}{8}} C^{\frac{3}{4}} .
$$

Solving this system, we obtain in the original parameters $\omega, \epsilon, \mu$ and $\sigma$ the asymptotic result

$$
p_{1}^{*}=\frac{(2 \omega \sigma \mu)^{\frac{1}{8}} C^{\frac{3}{4}}}{h^{\frac{3}{4}}}, \quad p_{2}^{*}=\frac{(2 \omega \sigma \mu)^{\frac{3}{8}} C^{\frac{1}{4}}}{2 h^{\frac{1}{4}}}, \quad \rho_{4}^{*}=1-\frac{(2 \omega \sigma \mu)^{\frac{1}{8}} h^{\frac{1}{4}}}{C^{\frac{1}{4}}} .
$$

The overlapping case. Also with overlap, $L=h$, the solution of of the min-max problem (5.16) equioscillates twice, and we find that $\left(p_{1}^{*}, p_{2}^{*}\right)$ is solution of

$$
\begin{aligned}
\rho_{4}\left(\bar{k}_{1}, \tilde{\omega}, \sigma, Z, L, p_{1}^{*}(1+i), p_{2}^{*}(1+i)\right) & =\rho_{4}\left(\bar{k}_{2}, \tilde{\omega}, \sigma, Z, L, p_{1}^{*}(1+i), p_{2}^{*}(1+i)\right) \\
& =\rho_{4}\left(\bar{k}_{3}, \tilde{\omega}, \sigma, Z, L, p_{1}^{*}(1+i), p_{2}^{*}(1+i)\right),
\end{aligned}
$$

where $\bar{k}_{1}, \bar{k}_{2}$ and $\bar{k}_{3}$ are three interior local maxima of $\rho_{4}$. For small $h$, we get

$$
\bar{k}_{1}=C_{b_{1}}, \quad \bar{k}_{2}=C_{b_{2}} h^{-\frac{2}{5}}, \quad \bar{k}_{3}=C_{b_{2}} h^{-\frac{4}{5}}, \quad p_{1}^{*}=C_{p} h^{-\frac{3}{5}},
$$

and solving the corresponding equations asymptotically, we find after a lengthy calculation for the constants the system

$$
C_{b_{1}}=\tilde{\omega}, \quad C_{b_{2}}=\sqrt{2 C_{p} C_{q}}, \quad C_{b_{3}}=\sqrt{C_{p}}, \quad C_{p}=\frac{4 C_{q}^{3}}{\tilde{\omega} \sigma Z}, \quad C_{q}=2 C_{p}^{2} .
$$

After its solution, we obtain in the original parameters $\omega, \epsilon, \mu$ and $\sigma$

$$
p_{1}^{*}=\frac{(\omega \sigma \mu)^{\frac{1}{5}}}{2 h^{\frac{3}{5}}}, \quad p_{2}^{*}=\frac{(\omega \sigma \mu)^{\frac{2}{5}}}{2 h^{\frac{1}{5}}}, \quad \rho_{4}^{*}=1-4 \sqrt{2}(\omega \sigma \mu)^{\frac{1}{10}} h^{\frac{1}{5}} .
$$

In Figure 5.2 we show again a comparison of the convergence factors. 
5.4. Optimization of the algorithm 5. We finally look for $s_{1}$ and $s_{2}$ of the form $s_{l}=p_{l}(1+i), l=1,2$ such that $p_{l}$ are solution of the min-max problem

$$
\min _{p_{1}, p_{2} \geq 0}\left(\max _{k \in K} \rho_{5}\left(k, \tilde{\omega}, \sigma, Z, L, p_{1}(1+i), p_{2}(1+i)\right)\right) \text {. }
$$

The non-overlapping case. We set $L=0, k_{\min }=0, k_{\max }=\frac{C}{h}$ and let $\left(p_{1}^{*}, p_{2}^{*}\right)$ be the solution of the min-max problem (5.16). We observe numerically that this solution equioscillates twice, where $\left(p_{1}^{*}, p_{2}^{*}\right)$ solves

$$
\begin{aligned}
\rho_{5}\left(\bar{k}_{1}, \tilde{\omega}, \sigma, Z, L, p_{1}^{*}(1+i), p_{2}^{*}(1+i)\right)= & \rho_{5}\left(\bar{k}_{2}, \tilde{\omega}, \sigma, Z, L, p_{1}^{*}(1+i), p_{2}^{*}(1+i)\right) \\
& =\rho_{5}\left(k_{\max }, \tilde{\omega}, \sigma, Z, L, p_{1}^{*}(1+i), p_{2}^{*}(1+i)\right),
\end{aligned}
$$

and $\bar{k}_{1}$ and $\bar{k}_{2}$ are two interior local maxima of $\rho_{5}$. Asymptotically, we obtain the behavior

$$
\bar{k}_{1}=C_{b_{1}} h^{-\frac{1}{13}}, \quad \bar{k}_{2}=C_{b_{2}} h^{-\frac{7}{13}}, \quad p_{1}^{*}=C_{p} h^{-\frac{10}{13}}, \quad p_{2}^{*}=C_{q} h^{-\frac{4}{13}} .
$$

Proceeding as in the other cases, we find for the constants the system

$$
C_{b_{1}}^{4}=6 C_{q} \tilde{\omega} \sigma Z, \quad C_{b_{2}}=\sqrt{2 C_{p} C_{q}}, \quad C_{q}=\frac{1}{2} \frac{C_{p}{ }^{3}}{C^{2}}, \quad C_{p}=\frac{\left(2^{8} \tilde{\omega}^{2} \sigma Z C^{10}\right)^{\frac{1}{13}}}{3^{\frac{3}{13}}},
$$

which allows us to find the asymptotic result in the original parameters to be

$p_{1}^{*}=\frac{2^{\frac{8}{13}} \omega^{\frac{2}{13}} \varepsilon^{\frac{1}{26}} \mu^{\frac{3}{26}} \sigma^{\frac{1}{13}} C^{\frac{10}{13}}}{3^{\frac{3}{13}} h^{\frac{10}{13}}}, p_{2}^{*}=\frac{2^{\frac{11}{13}} \omega^{\frac{6}{13}} \sigma^{\frac{3}{13}} \varepsilon^{\frac{3}{26}} \mu^{\frac{9}{26}} C^{\frac{4}{13}}}{3^{\frac{9}{13}} h^{\frac{4}{13}}}, \rho_{5}^{*}=1-\frac{2^{\frac{34}{13}} \omega^{\frac{2}{13}} \sigma^{\frac{1}{13}} \varepsilon^{\frac{1}{26}} \mu^{\frac{3}{26}} h^{\frac{3}{13}}}{3^{\frac{3}{13}} C^{\frac{3}{13}}}$.

The overlapping case. We finally treat the overlapping case $L=h$, where the solution of (5.18) equioscillates twice,

$$
\begin{aligned}
\rho_{5}\left(\bar{k}_{1}, \tilde{\omega}, \sigma, Z, L, p_{1}^{*}(1+i), p_{2}^{*}(1+i)\right)= & \rho_{5}\left(\bar{k}_{2}, \tilde{\omega}, \sigma, Z, L, p_{1}^{*}(1+i), p_{2}^{*}(1+i)\right) \\
& =\rho_{5}\left(\bar{k}_{3}, \tilde{\omega}, \sigma, Z, L, p_{1}^{*}(1+i), p_{2}^{*}(1+i)\right),
\end{aligned}
$$

and $\bar{k}_{1}, \bar{k}_{2}$ and $\bar{k}_{3}$ are three interior local maxima of $\rho_{5}$. Proceeding as before, we arrive after a long asymptotic analysis at

$$
p_{1}^{*}=\frac{\omega^{\frac{1}{4}} \varepsilon^{\frac{1}{16}} \mu^{\frac{3}{16}} \sigma^{\frac{1}{8}}}{2^{\frac{1}{4}} 3^{\frac{3}{8}} h^{\frac{5}{8}}}, \quad p_{2}^{*}=\frac{\sqrt{2} \sqrt{\omega} \varepsilon^{\frac{1}{8}} \mu^{\frac{3}{8}} \sigma^{\frac{1}{4}}}{3^{\frac{3}{4}} h^{\frac{1}{4}}}, \quad \rho_{5}^{*}=1-\frac{2^{\frac{23}{8}} \omega^{\frac{1}{8}} \varepsilon^{\frac{1}{32}} \mu^{\frac{3}{32}} \sigma^{\frac{1}{16}} h^{\frac{3}{16}}}{3^{\frac{3}{16}}}
$$

In Figure 5.2 we show the convergence factor in the non-overlapping and overlapping cases for the algorithm 5 compared to the classical one, algorithm 2, algorithm 3 and algorithm 4. Table 5.1 summarizes the asymptotic results that have been obtained for all algorithms.

6. Numerical results. We present here some numerical experiments in order to illustrate the performance of the optimized algorithms developed in the previous sections. The domain $\Omega$ is partitioned into several subdomains $\Omega_{j}$. The Maxwell's equations are approximated by a discontinuous Galerkin method $\left(D G-\mathbb{P}_{p}\right)$, see [12] for further details. In short, given a mesh $\mathcal{T}_{h}$ of $\Omega_{j}$ such that $\bar{\Omega}_{j}=\bigcup_{\tau_{k} \in \mathcal{T}_{h}}$, the $\left(D G-\mathbb{P}_{p}\right)$ method consists in searching an approximate solution of each field component in the set

$$
\begin{aligned}
& V_{h}=\left\{V \in L^{2}(\Omega) / \forall \tau_{k} \in \mathcal{T}_{h}, V_{\mid \tau_{k}} \in \mathbb{P}_{p}\left[\tau_{k}\right]\right\} \\
& \left.\mathbb{P}_{p}\left[\tau_{k}\right]=\text { polynomial function on } \tau_{k} \text { of degree } \leq p\right\}
\end{aligned}
$$



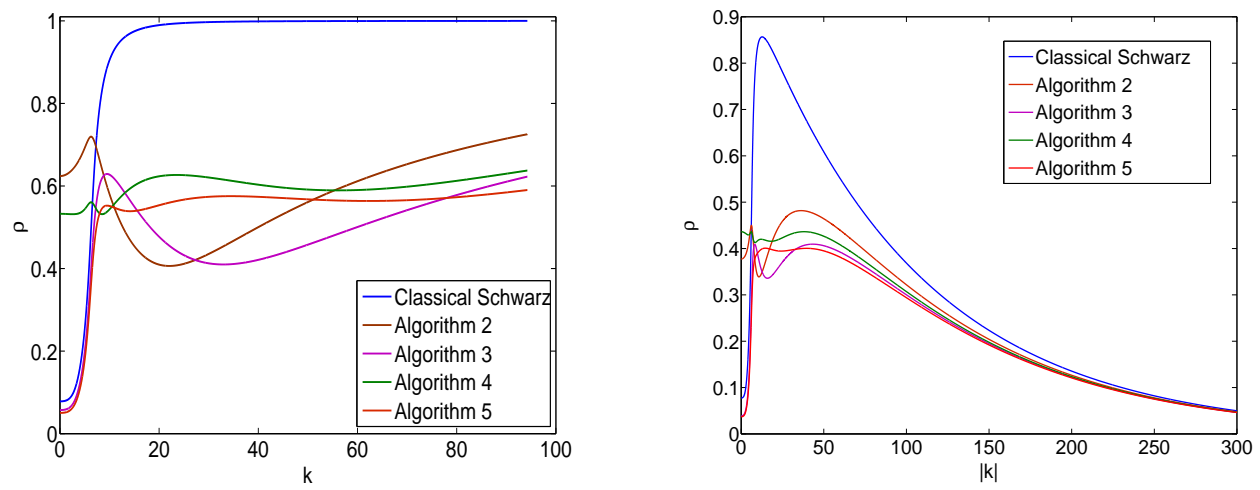

FIG. 5.2. Convergence factor comparisons of different algorithms for $\omega=2 \pi, \sigma=2$ and $\mu=\varepsilon=1$, for both non-overlapping $\left(L=0\right.$ ) (left figure) and overlapping $\left(L=h=\frac{1}{30}\right.$ ) (right figure) cases

\begin{tabular}{|c|c|c|}
\hline & \multicolumn{2}{|c|}{ with overlap, $L=h$} \\
\hline Algorithm & $\rho$ & parameters \\
\hline 1 & $1-\frac{4}{3}\left(9 \omega^{4} \sigma^{2} \mu^{3} \varepsilon\right)^{\frac{1}{8}} h^{\frac{3}{4}}$ & none \\
\hline 2 & $1-2^{\frac{7}{6}}(\omega \sigma \mu)^{1 / 6} h^{\frac{1}{3}}$ & $p=\frac{(2 \omega \sigma \mu)^{1 / 3}}{2 h^{\frac{1}{3}}}$ \\
\hline 3 & $1-\frac{2^{\frac{17}{10}}\left(\omega^{4} \varepsilon \mu^{3} \sigma^{2}\right)^{\frac{1}{20}} h^{\frac{3}{10}}}{3 \frac{3}{10}}$ & $p=\frac{2^{\frac{2}{5}}\left(\omega^{4} \varepsilon \mu^{3} \sigma^{2}\right)^{\frac{1}{10}}}{3^{\frac{3}{5}} h^{\frac{2}{5}}}$ \\
\hline 4 & $1-4 \sqrt{2}(\omega \sigma \mu)^{\frac{1}{10}} h^{\frac{1}{5}}$ & $p_{1}=\frac{(\omega \sigma \mu)^{\frac{1}{5}}}{2 h^{\frac{3}{5}}}, p_{2}=\frac{(\omega \sigma \mu)^{\frac{2}{5}}}{2 h^{\frac{1}{5}}}$ \\
\hline 5 & $1-\frac{2^{\frac{23}{8}}\left(\omega^{4} \varepsilon \mu^{3} \sigma^{2}\right)^{\frac{1}{32}} h^{\frac{3}{16}}}{3^{\frac{3}{16}}}$ & $p_{1}=\frac{\left(\omega^{4} \varepsilon \mu^{3} \sigma^{2}\right)^{\frac{1}{16}}}{2^{\frac{1}{4}} 3^{\frac{3}{8}} h^{\frac{5}{8}}}, p_{2}=\frac{\sqrt{2}\left(\omega^{4} \varepsilon \mu^{3} \sigma^{2}\right)^{\frac{1}{8}}}{3^{\frac{3}{4}} h^{\frac{1}{4}}}$ \\
\hline 1 & $1-\frac{\omega^{2} \sigma \sqrt{ }^{3} \varepsilon}{C^{3}} h^{3}$ & without overlap, $L=0$ \\
\hline 2 & $1-\frac{2^{\frac{3}{4}}(\omega \sigma \mu)^{\frac{1}{4}} \sqrt{h}}{\sqrt{C}}$ & $p=\frac{(\omega \sigma \mu)^{\frac{1}{4}} \sqrt{C}}{2^{\frac{1}{4}} \sqrt{h}}$ \\
\hline 3 & $1-\frac{2^{\frac{11}{7}}\left(\omega^{4} \varepsilon \mu^{3} \sigma^{2}\right)^{\frac{1}{14}} h^{\frac{3}{7}}}{3^{\frac{3}{7}} C^{\frac{3}{7}}}$ & $p=\frac{2^{\frac{4}{7}}\left(\omega^{4} \varepsilon \mu^{3} \sigma^{2}\right)^{\frac{1}{14}} C^{\frac{4}{7}}}{3^{\frac{3}{7}} h^{\frac{4}{7}}}$ \\
\hline 4 & $1-\frac{(2 \omega \sigma \mu)^{\frac{1}{8}} h^{\frac{1}{4}}}{C^{\frac{1}{4}}}$ & $p_{1}=\frac{(2 \omega \sigma \mu)^{\frac{1}{8}} C^{\frac{3}{4}}}{h^{\frac{3}{4}}}, p_{2}=\frac{(2 \omega \sigma \mu)^{3 / 8} C^{1 / 4}}{2 h^{\frac{1}{4}}}$ \\
\hline 5 & $1-\frac{2^{\frac{34}{13}}\left(\omega^{4} \varepsilon \mu^{3} \sigma^{2}\right)^{\frac{1}{26}} h^{\frac{3}{13}}}{3 \frac{3}{13}^{\frac{3}{13}}}$ & $p_{1}=\frac{2^{\frac{8}{13}}\left(\omega^{4} \varepsilon \mu^{3} \sigma^{2}\right)^{\frac{1}{26}} C^{\frac{10}{13}}}{3^{\frac{3}{13}} h^{\frac{10}{13}}}, p_{2}=\frac{2^{\frac{11}{13}}\left(\omega^{4} \varepsilon \mu^{3} \sigma^{2}\right)^{\frac{3}{26}} C^{\frac{4}{13}}}{3^{\frac{9}{13}} h^{\frac{4}{13}}}$ \\
\hline
\end{tabular}
ditions.

6.1. Performance for a two subdomain decomposition. We first test the propagation of a plane wave in a homogeneous and conductor medium using a TM formulation. The domain is $\Omega=(0,1)^{2}$, and the parameters are constant in $\Omega$, with $\varepsilon=\mu=1, \sigma=5$ and $\omega=2 \pi$. We impose on the boundary an incident field $\boldsymbol{W}^{i n c}=$ $\left(H_{x}^{i n c}, H_{y}^{i n c}, E_{z}^{i n c}\right)=\left(\frac{k_{y}}{\mu \omega}, \frac{-k_{x}}{\mu \omega}, 1\right) e^{-i \boldsymbol{k} \cdot \boldsymbol{x}}$ with $\boldsymbol{k}=\left(k_{x}, k_{y}\right)=\left(\omega \sqrt{\varepsilon-i \frac{\sigma}{\omega}}, 0\right), \boldsymbol{x}=$ $(x, y)$. The domain $\Omega$ is decomposed into two subdomains $\Omega_{1}=(0,1 / 2+L) \times(0,1)$ and $\Omega_{2}=(1 / 2,1) \times(0,1) ; L$ is the size of the overlap and is equal to the mesh size $h$ for the methods with overlap and is equal to zero for the methods without overlap. 

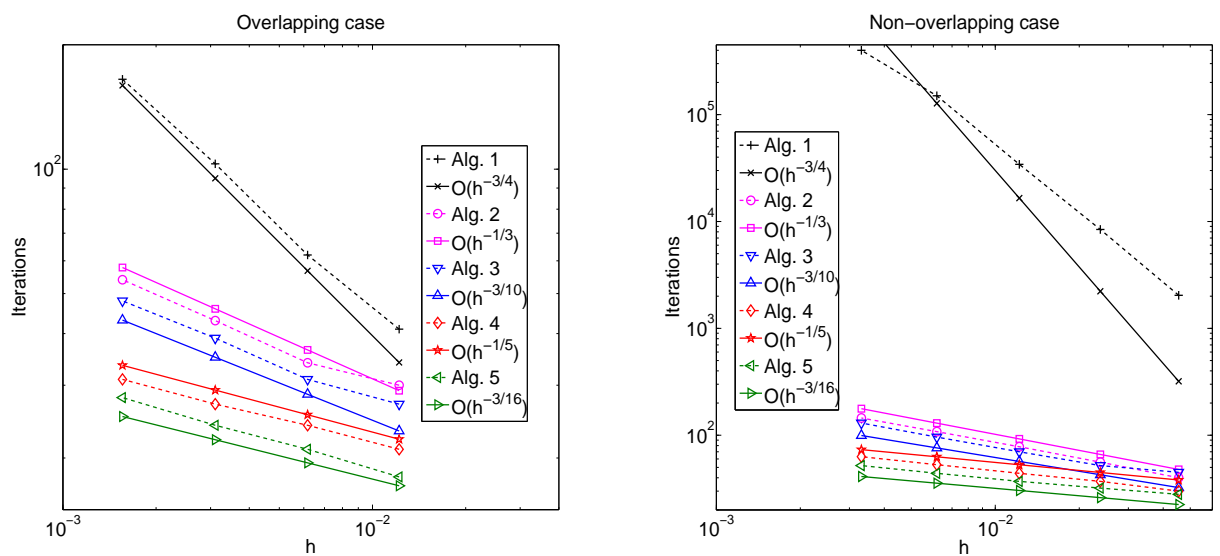

FIG. 6.1. Number of iterations against the mesh size $h$, to attain a relative residual reduction of $10^{-8}$

\begin{tabular}{|c|c|c|c|c|}
\hline Number of subdomains and processors & 2 & 4 & 8 & 16 \\
\hline \hline Algorithm 1 & 59 & 72 & 80 & 85 \\
\hline Algorithm 2 & 39 & 47 & 53 & 61 \\
\hline Algorithm 3 & 37 & 48 & 51 & 58 \\
\hline Algorithm 4 & 35 & 41 & 49 & 57 \\
\hline Algorithm 5 & 33 & 40 & 47 & 54 \\
\hline \multicolumn{4}{|l|}{}
\end{tabular}

TABLE 6.1

Iteration number comparison for the wave propagation problem in a subsurface

For this test, we use a $D G-\mathbb{P}_{2}$ method, i.e. a uniform polynomial approximation of order two. The performance of the algorithms is shown in Figure 6.1. In this figure, we show the number of iterations as a function of the mesh size $h$ for both the non-overlapping and overlapping cases. We have also represented in this figure the theoretical asymptotic results denoted by $O\left(h^{\alpha}\right)$. The numerical results are in good agreement with the theoretical asymptotic results which are summarized in Table 5.1, except for algorithm 1 (the classical algorithm) without overlap where we get better results. The fact that the discretization can improve the performance of Schwarz algorithms has also been observed and explained in [7] for Cauchy-Riemann equations.

6.2. A more realistic application. We present now a second more realistic test which consists in the simulation of electromagnetic wave propagation in a heterogenous subsurface medium. This kind of simulation is very important in imaging, see [13] for details. The configuration of the subsurface is shown in Figure 6.2 and is constituted of media which are characterized by various parameter values $\varepsilon$ and $\sigma$, $\varepsilon=1.5,2.25,3.25,4.25$ and $\sigma=10^{-6}, 10^{-5}, 10^{-4} 10^{-3}$. The computational domain is decomposed into several subdomains without overlap (a decomposition into eight subdomains is shown for example in Figure 6.3). We test here the performance of the algorithms for a decomposition into two, four, eight and sixteen subdmains. In Table 6.1, we have summarized the number of iterations needed for convergence, i.e to attain a relative residual of $10^{-8}$, depending on the number of subdomains. These 


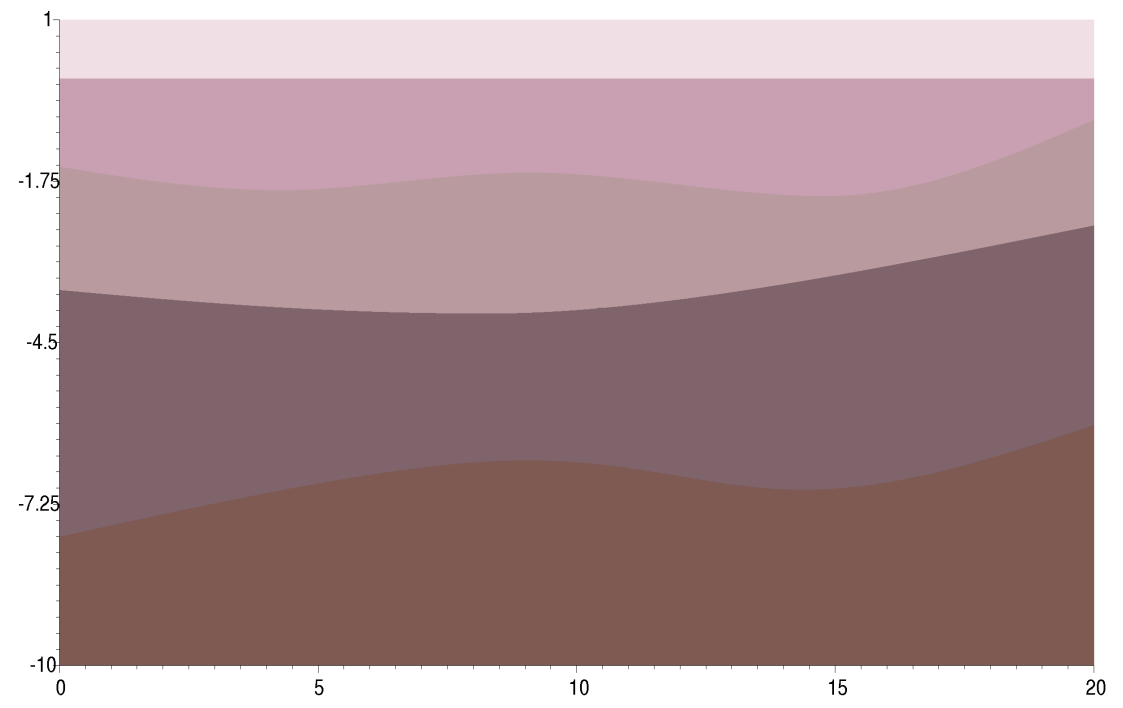

FIG. 6.2. Configuration of a subsurface

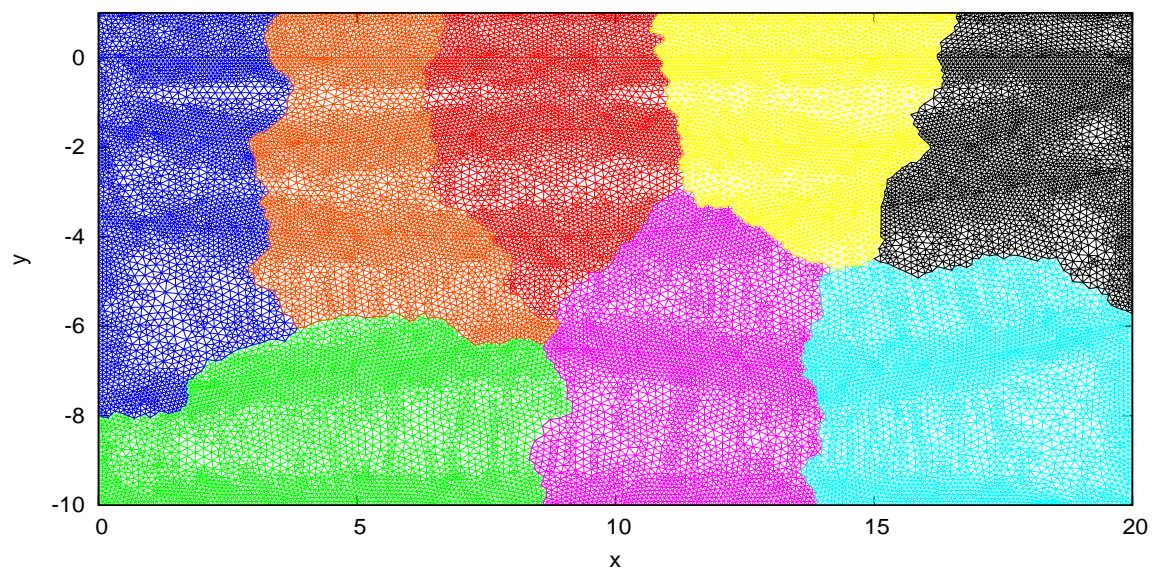

FIG. 6.3. Mesh and decomposition in 8 subdomains

results show that the optimized algorithms converge much faster than the classical algorithm (i.e. algorithm 1). We can also see that the hierarchy, in terms of number of iterations, of the optimized algorithms is respected as predicted by the theoretical results in Corollary 4.1. 
7. Conclusions. We have developed several domain decomposition algorithms based on optimized transmission conditions for Maxwell's equations with non-zero electric conductivity. Two algorithms are obtained by approximating the transparent operator by zeroth order transmission conditions while two further algorithms are based on second order transmission conditions. We have shown that the convergence factor of each algorithm can be written as $1-O\left(h^{\alpha_{i}}\right), i=1 . .5$. Our results are well confirmed by the first numerical test with a decomposition into two subdomains where we obtained also numerical convergence factors in the form $1-O\left(h^{\beta_{i}}\right), i=1 . .4$, with $\alpha_{i} \approx \beta_{i}$. The first test shows also that optimized Schwarz algorithms converge much faster than the classical one. This is also confirmed by the second more realistic test which shows also that these optimized algorithms can be effective for more complex problems and for arbitrary decompositions.

\section{REFERENCES}

[1] A. Alonso-Rodriguez and L. Gerardo-Giorda. New nonoverlapping domain decomposition methods for the harmonic Maxwell system. SIAM J. Sci. Comput., 28(1):102-122, 2006.

[2] I. Babuska and S. Sauter. Is the pollution effect of the fem avoidable for the Helmholtz equation considering high wave numbers? SIAM J. Numer. Anal., 34(6):2392-2423, 1997.

[3] P. Chevalier and F. Nataf. An OO2 (Optimized Order 2) method for the Helmholtz and Maxwell equations. In 10th International Conference on Domain Decomposition Methods in Science and in Engineering, pages 400-407, Boulder, Colorado, USA, 1997. AMS.

[4] P. Collino, G. Delbue, P. Joly, and A. Piacentini. A new interface condition in the nonoverlapping domain decomposition for the Maxwell equations. Comput. Methods Appl. Mech. Engrg., 148:195-207, 1997.

[5] B. Després. Décomposition de domaine et problème de Helmholtz. C.R. Acad. Sci. Paris, $1(6): 313-316,1990$.

[6] B. Després, P. Joly, and J. Roberts. A domain decomposition method for the harmonic Maxwell equations. In Iterative methods in linear algebra, pages 475-484, Amsterdam, 1992. NorthHolland.

[7] V. Dolean and M. J. Gander. Can the Discretization Modify the Performance of Schwarz Methods? In Domain Decomposition Methods in Science and Engineering XIX, 2010. submitted.

[8] V. Dolean, L. Gerardo-Giorda, and M. J. Gander. Optimized Schwarz methods for Maxwell equations. SIAM J. Scient. Comp., 31(3):2193-2213, 2009.

[9] V. Dolean, S. Lanteri, and R. Perrussel. A domain decomposition method for solving the three-dimensional time-harmonic Maxwell equations discretized by discontinuous Galerkin methods. J. Comput. Phys., 227(3):2044-2072, 2008.

[10] V. Dolean, S. Lanteri, and R. Perrussel. Optimized Schwarz algorithms for solving timeharmonic Maxwell's equations discretized by a discontinuous Galerkin method. IEEE. Trans. Magn., 44(6):954-957, 2008.

[11] M. El Bouajaji, V. Dolean, M. J. Gander, and S. Lanteri. Comparison of a one and two parameter family of transmission conditions for Maxwells equations with damping. In Domain Decomposition Methods in Science and Engineering XX, 2011. submitted.

[12] M. El Bouajaji, V. Dolean, M. J. Gander, S. Lanteri, and R. Perrussel. Domain decomposition methods for electromagnetic wave propagation problems in heterogeneous media and complex domains. In Domain Decomposition Methods in Science and Engineering XIX, 2010. submitted.

[13] M. El Bouajaji, S. Lanteri, and M. Yedlin. Discountinuous Galerkin frequency domain forward modeling dor the inversion of electric permittivity in 2d case. Geophysical Prospecting, in press, 2011.

[14] M. J. Gander, L. Halpern, and F. Magoulès. An optimized Schwarz method with two-sided Robin transmission conditions for the Helmholtz equation. Int. J. for Num. Meth. in Fluids, 55(2):163-175, 2007.

[15] M. J. Gander, F. Magoulès, and F. Nataf. Optimized Schwarz methods without overlap for the Helmholtz equation. SIAM J. Sci. Comput., 24(1):38-60, 2002.

[16] S.-C. Lee, M. Vouvakis, and J.-F. Lee. A non-overlapping domain decomposition method with non-matching grids for modeling large finite antenna arrays. J. Comput. Phys., 203(1):1- 
21, 2005.

[17] Z. Peng and J.-F. Lee. Non-conformal domain decomposition method with second-order transmission conditions for time-harmonic electromagnetics. J. Comput. Phys., 229(16):56155629, 2010.

[18] Z. Peng, V. Rawat, and J.-F. Lee. One way domain decomposition method with second order transmission conditions for solving electromagnetic wave problems. J. Comput. Phys., 229(4):1181-1197, 2010. 